\title{
Listening to mothers: qualitative studies on motherhood and depression from Goa, India
}

\author{
Merlyn Rodrigues ${ }^{\mathrm{a}}$, Vikram Patel ${ }^{\mathrm{a}, \mathrm{b}, *}$, Surinder Jaswal ${ }^{\mathrm{c}}$, Nandita de Souza ${ }^{\mathrm{a}}$ \\ a Sangath Society for Child Development \& Family Guidance, Goa, India \\ ${ }^{\mathrm{b}}$ London School of Hygiene \& Tropical Medicine, London, UK \\ ${ }^{\mathrm{c}}$ Tata Institute of Social Sciences, Mumbai, India
}

\begin{abstract}
There is little qualitative research on depression in motherhood from non-Western societies. The objective of the study described in this paper was to use qualitative methods to investigate the cultural validity of the construct of postnatal depression (PND) and its social and cultural contexts. The study was nested in a cohort of mothers recruited to study the risk factors and outcome of PND in Goa, India. In-depth interviews were carried out with 39 mothers (19 of whom were found to be suffering from PND as defined by a cut-off score on the Edinburgh PND scale) and their husbands purposively recruited from the cohort. An illness narrative was conducted with mothers who were categorized as suffering from PND and their husbands to elicit their explanatory models. The two groups (PND and non-PND) of mothers were comparable in terms of socio-demographic characteristics. PND mothers had lower levels of practical help and emotional support. The symptoms reported by PND mothers were similar to those recorded in studies with women in other cultures suggesting a universal clinical presentation of PND. Causal attributions for the experience of depression focused on economic difficulties and poor marital relationship. All mothers expressed the need for more practical help and support during the period after childbirth; husbands in both groups were often disengaged from baby care or supporting the mother. The study provides validity for the construct of PND in an Indian setting, but also shows that the emotional distress is interpreted from the context of social adversity, poor marital relationships and cultural attitudes towards gender rather than a biomedical psychiatric category. Contrary to the assumption that sociocultural contexts associated with childbirth in non-Western societies protect mothers from depression, factors unique to the culture such as gender preference and the low involvement of husbands in child-care are major causes of stress to mothers.
\end{abstract}

(C) 2003 Elsevier Science Ltd. All rights reserved.

Keywords: Post-natal depression; Cross-cultural; Social support; Maternal; India

\section{Introduction}

Qualitative research is recognized as a significant research strategy for determining women's health needs and appropriate interventions in South Asia (Gittelsohn et al., 1994). Although there is a growing body of epidemiological data on post-natal depression (PND) from developing countries, there is little qualitative

*Corresponding author. Sangath Centre, 71 Defense Colony Alto Porvorim, Goa 403531, India. Fax: +91-832-21-76-21.

E-mail address: vikpat@goatelecom.com (V. Patel). research on motherhood and depression. Three recent reviews on cross-cultural aspects of PND published in the 1990s were devoid of any relevant references (Howard, 1993; Kumar, 1994; Cox, 1996). However, there is a significant literature derived from anthropological research on the experience of motherhood in developing countries, including India (see Ram, 1998; Nichter \& Nichter, 1996). These studies have demonstrated the influence of such cultural factors as the perceived responsibility of the woman in bearing children, the value placed on the gender of the child, and the impact of the family structure and economic 
circumstances on the cultural construction of motherhood. Even though some anthropologists have argued that culturally specific rituals and factors associated with childbirth in non-Western societies protect mothers from depression (Stern \& Kruckman, 1983), there is little recent evidence to support this association.

There are concerns that, by using interviews and constructs developed largely in Western cultures for non-Western cultures, identifying "cases" of depression may constitute a category fallacy (Kleinman, 1987; Jadhav, 2000). The authors of this paper have been engaged in research on depression in non-psychiatric settings in Goa and Mumbai for many years. Their work with general adult populations has shown that depression can be identified reliably using psychiatric interviews, provided adequate attention is given to a variety of translation issues so that conceptual equivalence of items and the use of local idioms of distress are given due regard (Patel, Pereira, \& Mann, 1998). The present study is related to epidemiological investigations of depression in motherhood during which a short measure of PND, the Edinburgh PND Scale, was translated and validated for use in the Konkani language of Goa (Patel, Rodrigues, \& De Souza, 2002). The EPDS has a history of use in many different cultures, including elsewhere in India (Cox \& Holden, 1996). The epidemiological study demonstrated that mothers whom the EPDS classified as a case of PND were significantly more likely to be functionally impaired and to seek health care than other mothers, a measure of convergent validity of the construct. This study had also demonstrated that gender-based variables and social adversity were major risk factors for PND.

The study described in this paper aimed to describe attitudes and perceptions of mothers and husbands towards childbirth in Goa in order to explore the processes through which the relationship between social adversity and PND is mediated. The second objective was to describe the explanatory models of PND with the aim of studying the cultural validity of the construct of PND.

\section{Method}

\section{Setting}

Goa is the smallest state in India and has a population of 1.4 million. The crude birth rate estimated in 1992 was 14.5 per 1000 population. Most births $(>90 \%)$ are attended at delivery by trained personnel (International Institute for Population Sciences, 2001). Literacy rates are relatively high in Goa, with $89 \%$ males and $75 \%$ females being literate. The most widely spoken language is Konkani.

\section{Sample}

The sample was recruited purposively from a cohort study of PND conducted in a district hospital in north Goa (Patel et al., 2002). A cohort of 270 women attending the antenatal clinic was recruited. Fifty-nine out of 252 who were reviewed at $6 / 8$ weeks post-natally were identified to be cases of PND based on a validated cut-off score of 11/12 on the EPDS. From the sample of 252 women, 39 ( 19 women with and 20 without PND) who were willing to participate, spoke either Konkani or English, and were articulate were purposively selected for the qualitative study. All the respondents selected were assured that participation was voluntary and that no information identifying the individuals would appear in reports. We will refer to the two groups of mothers as the "PND mothers" and "non-PND mothers".

\section{Data collection}

The method of data collection was in-depth interviews with mothers and husbands. The interviews were scheduled in the month after the 6/8-week post-natal review. Only one interviewer (MR) conducted all the interviews to rule out discrepancies in the themes discussed and style of interviewing. This interviewer had been trained in qualitative methods by two authors (SJ \& VP) who had considerable experience in qualitative methodologies (Blue, Ducci, Jaswal, Ludermir, \& Harpham, 1995; Patel \& Prince, 2001). Interviews were conducted in the woman's home after obtaining consent and making an appointment for a convenient time. Interviews were recorded with a tape recorder. All respondents were interviewed individually and privacy was maintained during the interview. Husbands were interviewed separately. Each interview was conducted in one sitting and the average duration was approximately $45 \mathrm{~min}$. Husband interviews usually lasted for a shorter period. Interviewing techniques, such as free listing and ranking, were used to aid in data collection (Jaswal and Harpham, 1997). The themes used for the in-depth interviews were health problems associated with childbirth and support systems available to the mother focusing on practical support, emotional support, and rituals associated with childbearing. An illness narrative was conducted only with PND mothers and their husbands and explored the perceived cause, symptoms, impact, and help-seeking for PND.

\section{Data analysis}

The recorded interview was transcribed verbatim into the language of the interview (all but two interviews were in Konkani) and then translated into English. Categories for analysis of data were the themes identified at the outset. The transcripts were analysed 
in an iterative fashion by all four authors to achieve consensus on the allocation of transcript material from each subject to various categories. Data were then analysed according to these categories. Issues that recurred between subjects were of particular interest because of their potential for generalizability. Analysis of data was divided into four groups: husbands and mothers separately for the two groups of mothers.

\section{Results}

Socio-demographic and clinical data on the 39 mothers is shown in Table 1. We were able to interview 27 husbands. The remainder were not available due to their work schedules; for example, some husbands' work-place was so far from their marital home that they stayed in a room somewhere near the work place for weeks.

\section{Illness associated with childbirth}

Mothers were asked to list all the illnesses they associated with childbirth. Table 2 shows these illnesses, the frequency with which they were reported, and the ranking of their perceived severity. The commonest illness categories were symptoms such as lower backache, heavy menstrual flow, stomach ache, body ache, and colds. The PND mothers reported a high percentage of aches and pains, while the non-PND mothers most commonly reported heavy menstrual flow. Women in both groups hesitated to consult doctors for their illnesses, often perceiving them as minor, natural consequences of childbirth. They usually opted for home remedies like oil massages to relieve symptoms of aches and pains and cossai (a mixture of ginger and tulsi) for colds. Symptoms associated with psychiatric disorders were not volunteered as a health problem associated with childbirth by any of the PND mothers; however, three non-PND mothers described nervachem, a Konkani term used to describe stress-related emotional problems. As one woman described:

Some women get mental problems after delivery. Some women are weak, so they are prone to get nervous problems. Someone had it in our neighbourhood. After the birth of her child she started talking rubbish, got easily excited, would cry. She had given birth to a girl child. It happens soon after the delivery for some women. It happens if you had too many problems during delivery. It's not like they are possessed by an evil spirit. After delivery the person should not have tensions. We all have tensions but after delivery you should have peace, husband should be nice. Some husbands beat their wives therefore they have tensions.

In both groups, women ranked an illness, which was painful and affected their ability to work and provide care as more serious. The majority of PND mothers mentioned lower backache as the most severe illness because of its painful nature and impact on their daily routine, whereas most non-PND mothers considered emotional problems as the most severe because "the children are then neglected as the mother tends to be in a world of her own". Husbands rarely asked their wives about their health. Thus, many said that they did not take much interest in "women-related matters", or that they had not heard of any illnesses. Some husbands reported body aches, stomach pains, headaches, and pains associated with sutures, giddiness, weakness because of loss of blood, cold because of working in cold water, and fever as illnesses associated with childbirth.

\section{Causes of illnesses associated with childbirth}

Differences were noted in causal models between PND and non-PND mothers. Overwork and lack of practical support were the most common causal models for health problems in PND mothers, as compared to somatic causal models for non-PND mothers. For

Table 1

Sociodemographic and clinical characteristics of the sample

\begin{tabular}{lll}
\hline Variable & PND mothers $N=19$ & Normalmothers $=20$ \\
\hline Age, mean $(95 \% \mathrm{CI})$ & 25.5 years $(24.1-26.9)$ & 27.3 years $(25.2-29.3)$ \\
Not working outside the home & $18(95 \%)$ & $20(100 \%)$ \\
Living in a joint family & $11(58 \%)$ & $11(55 \%)$ \\
Literate & $19(100 \%)$ & $14(70 \%)$ \\
Primigravida & $6(32 \%)$ & $4(20 \%)$ \\
Rural residence & $14(73 \%)$ & $17(85 \%)$ \\
Mean EPDS Score $(95 \% \mathrm{CI})$ & $17.4(15.1-19.7)$ & $3.8(2-5.6)$ \\
Male gender of newborn & $54 \%(10)$ & $70 \%(14)$ \\
Husbands mean monthly income (s.d.) & Rs. 1805 (s.d. 644) & Rs. 2175 (s.d. 1089) \\
\hline
\end{tabular}


Table 2

Illnesses related to childbirth (arranged in descending order of frequency of reporting by the PND mothers)

\begin{tabular}{|c|c|c|c|c|c|}
\hline Local term & Description/illness & $\begin{array}{l}\text { Proportion of } \\
\text { PND women } \\
N=19\end{array}$ & $\begin{array}{l}\text { Number who } \\
\text { ranked the } \\
\text { illness as the } \\
\text { most severe }\end{array}$ & $\begin{array}{l}\text { Proportion } \\
\text { Non-PND } \\
\text { women } N=20\end{array}$ & $\begin{array}{l}\text { Number who } \\
\text { ranked the } \\
\text { illness as the } \\
\text { most severe }\end{array}$ \\
\hline Bendant dukta & $\begin{array}{l}\text { Lower back ache/hip } \\
\text { ache }\end{array}$ & $14(73 \%)$ & 10 & $4(20 \%)$ & 1 \\
\hline Angar foddta & Body ache & $11(57 \%)$ & 2 & $3(15 \%)$ & \\
\hline Potant dukta & Stomach ache & $9(47 \%)$ & 2 & $7(35 \%)$ & 3 \\
\hline Angar zavop & Heavy menstrual flow & $8(42 \%)$ & 1 & $9(45 \%)$ & 2 \\
\hline Thondi & Cold & $6(31 \%)$ & & $6(30 \%)$ & 1 \\
\hline Tokli foddta & Headaches & $5(26 \%)$ & & $2(10 \%)$ & \\
\hline Dhovem angar & White discharge & $3(15 \%)$ & & $4(20 \%)$ & 1 \\
\hline Ang bhair sorta & Vaginal prolapse & $3(15 \%)$ & & - & \\
\hline Thoin dukta & Vaginal pain & $3(15 \%)$ & & - & \\
\hline Nidh podona & Poor sleep & $2(10 \%)$ & & - & \\
\hline Zor & Fever & $2(10 \%)$ & & $3(15 \%)$ & 1 \\
\hline Markund & Abdominal pain & $1(5 \%)$ & & $5(25 \%)$ & 2 \\
\hline Nervachem zata & Nervous problems & - & & $3(15 \%)$ & 3 \\
\hline Ghuvol yeta & Giddiness, dizziness & $3(15 \%)$ & & $3(15 \%)$ & 1 \\
\hline Faat foddta & $\begin{array}{l}\text { Backache during } \\
\text { pregnancy. }\end{array}$ & - & & $3(15 \%)$ & \\
\hline Garmi zali ani fodd aile & Infected operative scar & - & & $3(15 \%)$ & 1 \\
\hline Lut martha & $\begin{array}{l}\text { Swelling of the body } \\
\text { during pregnancy }\end{array}$ & - & & $2(10 \%)$ & \\
\hline
\end{tabular}

example, a PND mother, explaining why backaches occur, said the following:

After delivery, the whole body tends to ache and therefore oil is massaged all over the body. But it wasn't done for me... I get backaches because I have to work. I don't get rest. There is no help from anyone. If I had rested then this wouldn't have happened. I have to stand, sit [most families usually have their fireplaces on the floor and hence have to squat while they cook meals]. Then I have to do work in water... I do try to massage myself, but I can't reach my own back!

A non-PND mother described body aches in this manner:

Sometimes I do get body aches, but I soon get relief and there is nothing else [no other complaint] so I didn't see a doctor. We know that blood becomes less and that's why I should take tonics and then I feel better. Doctor has prescribed the tonic and I still take it. I have slightly less blood.

Some mothers and fathers mentioned the gender of the baby as a possible cause for nervous problems; mothers also mentioned relationship problems with significant others as an antecedent of mental distress.

\section{Support and maternal well-being}

\section{Practical Support}

All women agreed that they received practical support in caring for the baby and doing other house-related work. Practical support was usually received from husbands, mothers, sisters, and an older child. Despite this, the majority expressed the need or desire for more support. For example, a first-time mother who had postponed the customary visit to her own mother's home in the post-natal period said:

I have to do everything by myself. I need someone to help me. I haven't gone to stay with my mother because then my husband will have trouble managing things; he will have to cook for himself. So I'll go later and stay a month with her.

There was a distinct difference in the nature of practical support received by the women in the two groups. Non-PND mothers reported help in tasks that are generally considered physically demanding and tiring, for example, fetching water and washing clothes. PND mothers, on the other hand, described their husbands playing with the baby when they returned home from work or helping in household chores only when they were asked to. Feeling overworked and unsupported was linked to tiredness and other 
symptoms, which in turn may have contributed to strained relationships. For example, one PND mother said:

I do most of my work with water. I get a cold because I wash clothes. After delivery one gets rest for at least three months but I had rest only for a month and from the next month onwards I had to do all the work, and that's the reason why I have all these health problems. ... The doctor cannot find anything. This is why I think I fall ill because of tension. I get a cold and then it passes on to my child and then he too falls ill... I have to wash clothes all by myself. I take a lot of time to do this, and after that I have to look after the child and by the time I finish doing everything it is 2.30 p.m. and by then my hunger dies. Before I used to enjoy everything, now I don't enjoy anything. I feel like there is a heavy burden on my head. I don't have the motivation to do anything... I don't do anything for myself because I am fed up of my life... my husband does help, but he has to be told!

Husbands of PND mothers were less aware of the need to offer practical help to their wives. The usual theme raised was their perceived need to provide financial support. Most acknowledged that their wives did most of the work, though a few mentioned that, during the first few weeks after delivery, they did more household chores than usual. In terms of receiving support themselves, more than half the husbands in both groups described practical support from their brothers, in laws, and their own mothers.

\section{Emotional support}

Both groups of mothers reported a similar degree of emotional support from their husbands and mothers. However, only PND mothers reported a difficulty in sharing feelings. As one mother said, "I feel if I talk to somebody, I will feel better. But this will also lead to quarrels, so it is best not to talk to anybody". PND mothers also spoke of strained relationships with their husbands and in-laws because of the gender of the baby. Three mothers spoke of physical abuse. As one woman commented:

He used to hit me when I was pregnant. We had an argument and I said I was going to leave him. He said we should both die and save our lives. I said I would give back all the money he had spent on me. Then, he hit me so badly; I fell on my stomach. The baby did not move for an hour and I thought it had died. He has stopped hitting now, but sometimes he gets irritated and says "I will hit you" and I get scared.

Whereas nearly two-thirds of PND mothers expressed the need for more emotional support, all non-PND mothers spoke of being satisfied with the levels of emotional support they received. They reported having a husband, friend, neighbour, or a mother with whom to share their feelings. Even though some non-PND mothers indicated that they did not like to share their feelings for fear of everyone knowing their problems, they did manage to speak to their husbands or mothers, unlike the PND mothers, who kept things to themselves. One PND mother said: "I don't confide my feelings to anybody, I don't need anyone. I feel like committing suicide". Husbands too described difficulty in expressing feelings but had more opportunities to share.

\section{Rituals of childbearing}

Several rituals are performed to celebrate the birth and specific practices carried out to maintain good health for the mother and baby. There were slight variations in the rituals according to the baby's genderfor example, jellabis, a less expensive sweet, were shared if the baby were female, instead of pedhas, a comparatively expensive sweet, for male babies. Notably, there were no significant differences in the rituals and practices (for the mother or the baby) reported by the two groups. An exception was oil massage for the mothers. This popular custom is believed to improve strength and maintain general health in mothers and their babies. However, eight (42\%) of the PND mothers said that this had not been done for their current pregnancy. Similarly, a lesser number of PND mothers reported being given a special diet in the period following childbirth, as compared to non-PND mothers, who spoke of being given nourishment to increase breast milk, reduce body pain, and improve strength.

\section{Explanatory models of PND mothers and husbands}

\section{Symptoms and impact}

Symptoms reported by mothers are shown in Table 3. Most of the mothers felt that their symptoms had an adverse impact on their relationship with their baby, significant others, and in the performance of household tasks. For example, even though the overriding emotion towards the baby was that of happiness and joy, on further probing, nearly half the mothers (42\%) mentioned anger and irritability which adversely affected relationships with their spouse and children. One mother said, "When they [the children] come home from school, I get fed up and hit them". Many women also described the impact their emotional state had on household chores. One said, "When there is tension, I do just the opposite of what I have to do because I am lost in my own thoughts". Some mentioned lack of motivation to do any work, thus leaving many necessary chores unfinished and neglected. None of the mothers were able to label this distress; none perceived this in the context of a biomedical psychiatric disorder. 
Table 3

Symptoms reported by PND mothers and their husbands

\begin{tabular}{|c|c|c|}
\hline Symptom & Proportion of mothers (numbers) & Proportion of husbands \\
\hline Headaches & $13(68 \%)$ & $1(7 \%)$ \\
\hline Sleep problems & $11(57 \%)$ & $1(7 \%)$ \\
\hline Worries/think a lot & $11(57 \%)$ & \\
\hline Crying & $10(52 \%)$ & $2(14 \%)$ \\
\hline Giddy/dizziness & $6(31 \%)$ & $1(7 \%)$ \\
\hline Self harm thoughts & $5(26 \%)$ & \\
\hline "Tension", nervousness & $5(26 \%)$ & \\
\hline Not wanting anything. No interest & $5(26 \%)$ & $3(21 \%)$ \\
\hline Tired/weak & $4(21 \%)$ & \\
\hline Loss of appetite & $3(15 \%)$ & \\
\hline "Feel strange", uneasy, faint & $3(15 \%)$ & \\
\hline Irritable & $3(15 \%)$ & $1(7 \%)$ \\
\hline Sadness & $2(10 \%)$ & $2(14 \%)$ \\
\hline Body aches & $2(10 \%)$ & $1(7 \%)$ \\
\hline
\end{tabular}

The following dialogue illustrates the symptoms and causal models of depression in one mother:

Interviewer- "Earlier, you said that you were tense and your head is not feeling alright; why do you have this tension?"

Mother- "Once you are married you are under the care of your husband. If your husband is not good then there is tension and you wonder why you ever got married. One needs to have at least five minutes of happiness, right?"

Interviewer- "How does this 'tension' affect your health?"

Mother- "The headaches, dizziness, cold, tiredness all are the effects of this tension".

Interviewer- "Among these that you have mentioned, which occurs most frequently?

Mother-"When one thinks too much, you get drowsy".

Interviewer-"What happens then?"

Mother-"Then you become weak and there is no appetite and so you can't eat or do anything for that matter".

Interviewer- "When you feel this way, who do you confide your feelings to?"

Mother-"I don't tell anybody".

Interviewer- "Do you feel you need somebody to talk to when you are sad?"

Mother-“I don't need anybody. I just feel like ending my life".

Few husbands were aware of the emotional or other symptoms in their wives but were unaware of how to respond, as illustrated in this dialogue.

Interviewer - "Do you feel this 'tension' that you have mentioned is a kind of an illness?"

Father-"Yes, I do feel it's an illness".

Interviewer- "Did your wife go to a doctor because of this?"
Father- "No, this is home tension, work [household work] tension".

Interviewer-"What type of home tension?"

Father-"I don't know".

Interviewer- "You do not know why she is like this but you think it is because of tension?"

Father-"Yes"

Interviewer- "How do you come to know she is not her usual self?"

Father- "She is quiet, she doesn't talk to anybody".

Interviewer- "Do you ask her why?"

Father -"What to ask? She doesn't respond!"

\section{Causal models}

Mothers volunteered several causes for their distress; however, most husbands were not aware that their wives were unwell in any specific or significant manner. Economic difficulties were the commonest causal model. As one mother said:

The main tension is because of the unemployment of my husband. Since it's summer now, he can go anywhere (and do odd jobs), but once it starts raining, where can he go... My elder son has jaundice. If one of them is better, then the other one falls ill. I'm worried because we have to buy the medicine prescribed by the doctor.

The next most common reason for distress was a poor relationship with significant others in the family, in particular, the husband and/or mother-in-law. Some mothers opined that the birth of a girl child contributed to their strained relationship with their husband and inlaws, as illustrated in this dialogue:

Interviewer- "What did you feel when you saw your baby after delivery?" 
Mother-" "I asked them [nurses] who is born [boy or girl]? They replied saying that they are not going to take away the baby. I answered, it's not because of that, but I want a son. They told me it makes no difference whether it's a boy or a girl and they began convincing me. They didn't tell me that the baby born was a girl at first but after they finished telling me that it doesn't matter if it's a boy or a girl, then they told me [the baby was a girl]". Interviewer-"What did you feel at this time?"

Mother-"I felt bad because everyone was expecting a son and since this did not happen I was thinking, "what will my people say!"”

Interviewer- "So it [whether it's a boy or a girl child] makes no difference to you?"

Mother-"I feel nothing of that sort but I was afraid because of them [in-laws]. It makes no difference to me because, after all, it's God's will. I am only afraid of them, of what they will say or what will they do to me".

\section{Treatment}

Mothers did not feel there was any value in informing their husbands of their distress because of the feeling that their husbands would be disinterested or even hostile, as evidenced by the following:

"What's the point in telling him my worries? What can he do? How can I tell him what I am going through? He is a man, after all."

"If I tell him anything, he gets irritated and tensed up. I don't talk to anyone and I don't like it if anyone comes to my house. That's why my sickness has become worse; because I don't express my feelings to anyone."

The majority of mothers were not taking any treatment for their distress despite recognizing that they felt unwell. The most frequent reasons were the perception of the lack of treatment options for their illness, and concern about the reaction from family members. For example, one mother said, "If I am ill, I have to ask my husband if I can go to see the doctor. If he says no, I cannot go. I feel hurt and I get thoughts of who will take care of my kids in case I die".

Of the three mothers who had sought medical treatment, none complained directly to the doctor about their emotional symptoms, choosing instead to complain about headaches and tiredness. One woman admitted to being hospitalized for these complaints. She expressed feelings of helplessness: "What is the use of taking any treatment? Whatever has to happen will happen. There's no solution for it. If he [husband] is good, then everything would be fine".

One woman mentioned that she would just sit at a table and cry for God's help. Several women (6) chose to distract themselves from their symptoms and worries by engaging in household work, looking after the children, watching TV, or by being in the company of others. Only two mothers denied the fact that they were ill and therefore did not feel the need to seek treatment.
Only four husbands considered medical intervention for their spouses, one of whom could not afford it. Two husbands applied balms or oil to ease headaches. One suggested cracking jokes to make the mother laugh. Four husbands refused to accept the fact that their wives suffered from any emotional problem.

\section{Discussion}

This paper describes a qualitative investigation aimed at understanding the mechanism through which the relationship between social adversity and PND is mediated, and the cultural validity of the biomedical category of PND. The study is, to the best of our knowledge, the first such study from South Asia. The integration of the qualitative research as part of a larger epidemiological investigation and the involvement of husbands as study subjects are methodological strengths of the study.

A key finding was that, despite the assumption that the social contexts of childbirth as a "rite of passage" provides mothers with protection from depression (Cox, 1996), stressors unique to the rapid changes in the local culture may increase mothers' vulnerability. Stern and Kruckman's (1983) review of the ethnographic literature on PND found that factors such as mandated rest, assistance from relatives and social recognition through rituals were key factors, which prevented PND. The failure to observe rituals and dietary practices associated with childbirth, such as the use of special diets perceived to be nutritious and body massages with oil, was associated with the experience of depression in our study. The relevance of such folk dietary models and rituals has been described by other authors in ethnographic studies of pregnancy in India (Nichter \& Nichter, 1996). Other authors have also described the growing influence of medical models in pregnancy and childbirth on women's experiences of motherhood: for example, in the context of the emphasis on hospitalbased deliveries in settings where births traditionally took place in one's home (Ram, 1998). Another significant change has been the rapid decline in fertility and family size as a result of the active campaign to limit population growth in India. Such rapid changes in health, social, and family structures in recent decades are potentially leading to a decrease in the culturally sanctioned practices, which traditionally supported mothers in pregnancy and childbirth. A visible example of these changes is in the declining proportion of mothers living in extended or joint families, in effect reducing the number of adults in the household who could have played a supportive role for mothers. Thus, as Indian families experience a change in their dynamic structure, mothers are left carrying more of the burden of care associated with childbirth. It is possible that 
these changes may, in part, account for the high rates of PND reported in recent studies from developing countries (Patel et al., 2002; Cooper et al., 1999).

It is not surprising, therefore, that mothers who were classified as suffering from PND revealed lower levels of practical support and poorer levels of emotional support when compared with other mothers. The vast majority of these mothers had never shared their suffering with their husbands, who remained unaware of their wives' emotional health. The quantitative investigation found a strong association between lack of social support, marital difficulties, and economic difficulties and the risk of PND; the qualitative investigation found that the most common causal attribution was a poor marital relationship and economic difficulties. These risk factors and causal attributions are similar to those reported from Western cultures and provide support from a nonWestern society for a psychosocial etiology for PND (Cooper \& Murray, 1998; Brugha et al., 1998; O'Hara \& Swain, 1996). A poor marital relationship expressed itself through inadequate practical and emotional support for the mother. Violence was cited by some mothers and represents the extreme example of marital disharmony. The influence of boy preference, much reported in South Asia (Winkvist \& Akhtar, 2000) and found to be a major effect modifier in the epidemiological investigation (Patel et al., 2002) was confirmed in the qualitative investigation. Boy preference was most marked for mothers who had living girl children and was often expressed through lack of support and hostility from the husband and mother-in-law. The girl child was cited as a heavy economic drain on the family. This was partly due to the perception that most girls would ultimately be married and would not contribute economically to the family and that dowry payments would need to be made at the time of marriage. The male, on the other hand would one day be an earning member of the family and also eventually gain a dowry. The findings of this study may be interpreted in the light of the work of Brown and Harris (1978) on the social origins of depression. Thus, the existence of a poor marital relationship may act as a vulnerability factor, which in the light of a provoking element such as the birth of a girl child, triggers a depressive episode. On the other hand, the birth of a boy may act as a protective factor even for mothers who live in an unhappy marital situation.

The second key finding is the validation of the clinical construct of PND. Depression, like all biomedical psychiatric categories, has its roots in a Western medical and cultural framework (Jadhav, 2000). The evolution of this category has been especially enhanced by the development of medications supposedly targeted to treat this disorder, a historical process which has been eloquently described by Healy (1997). The cross-cultural validity of the biomedical construct of depression is one essential way of examining its validity as a biomedical category. Two approaches were used by the authors to study the cultural validity of the category of PND, both of which were based on comparing mothers who a Western instrument categorised as suffering from PND with mothers who were not. First, the cohort study within which the qualitative investigation was nested demonstrated that PND mothers were significantly more disabled and more likely to consult health providers than other mothers (Patel et al., 2002).

These quantitative findings suggested convergent validity for the construct of PND. In the qualitative component described in this paper, we aimed to elicit the illness narratives through open-ended questions in the local language. The explanatory models elicited from mothers who had been classified as being depressed in the qualitative investigation revealed high rates of symptoms and idioms of distress, which were similar to those described in studies from Western societies. As mentioned earlier, these mothers also described causal models, which show close similarity to the etiological models for PND as described by mothers in Western cultures. Thus, mothers who were classified as cases of depression on the basis of a cut-off score on a psychiatric questionnaire identified a group who were functionally impaired and who experienced symptoms typically associated with emotional distress in other cultures. However, it was notable that none of these mothers (or their husbands) considered a psychiatric term for their suffering; only a minority, however, did not acknowledge that their distress was an illness. The lack of any local term which could be used to describe depression as a biomedical category and the lack of awareness amongst health care providers has been reported in earlier studies from Goa in primary care (Patel, Pereira, Coutinho, \& Fernandes, 1997). These findings lend support to the notion that the use of biomedical psychiatric labels may not be acceptable to many mothers and an alternative approach to recognition and management may be required. This may involve the use of symptom and context-based terms such as tension, weakness, and difficulties in one's relationships at home. Thus, although PND, as a biomedical construct comprising emotional and somatic distress in the setting of social adversity, may be crossculturally valid, its conceptualisation as a psychiatric, or even medical, disorder is less applicable across cultures. Instead, the experience of depression is interpreted in terms of socioeconomic and relationship difficulties. In this sense, depression is, "like any other disease: an interpreted disorder" (Good, Good, \& Moradi, 1985). The association of depression with social adversity does not, however, imply that acceptance of the biomedical construct is tantamount to "medicalising social distress"; thus, most persons who live in adversity do not develop a depressive disorder (Patel, Araya, Lewis, 
\& Swartz, 2001). A useful analogy is that of tuberculosis which is also associated with poverty and social adversity but is still recognized as a discrete biomedical disease. At the heart of the difference in the interpretation of the epidemiological findings of the association of social adversity with these conditions is the demonstrable pathology of tuberculosis and its effective pharmacological treatment, distinguishing it as a "disease" as opposed to the category of depression which remains an "illness"(Littlewood, 1991).

In conclusion, this study provides an insiders view of postnatal depression and points to the cultural validity of the syndrome of PND and the social processes through which some mothers in the cultural setting of Goa, India, experience stress and consequently develop a depressive illness. Thus, for some women, the postpartum period is a time of considerable stress, a situation characterized by the need for immense physical and emotional support. The implications of the study findings for future research are in the context of developing psychosocial interventions for prevention and management of PND. Any intervention will need to emphasize the husband's involvement in supporting the mother practically and emotionally, to identify and work on issues causing strain in the marital relationship, and in the case of extended families, the involvement of key family members such as mothers-in-law in the therapy. Tackling the deep-rooted culturally determined beliefs of son preference may be an important theme, particularly for mothers who already have living girl children.

\section{Acknowledgements}

The study was supported by a MacArthur Foundation Population Fellowship. We are grateful to the Directorate of Health Services for having permitted us to base the study in a district hospital in north Goa. We thank all the mothers and husbands who participated in the study. We are also grateful to Lucy Martins, Subodh Phadke, Joaquim Godinho, Livia Coutinho and Pacienca Cardozo for assistance with transcription and translation.

\section{References}

Blue, I., Ducci, M. E., Jaswal, S., Ludermir, A., \& Harpham, T. (1995). The mental health of low-income urban women: Case studies from Bombay, India; Olinda, Brazil; and Santiago, Chile. In T. Harpham, \& T. Blue (Eds.), Urbanization and mental health in developing countries (pp. 75-101). Aldershot: Avebury.

Brown, E., \& Harris, T. (1978). Social origins of depression. London: Tavistok.
Brugha, T. S., Sharp, H. M., Cooper, S. A., Weisender, C., Britto, D., Shinkwin, R., Sherrif, T., \& Kirwan, P. H. (1998). The Leicester 500 project: Social support and the development of postnatal depressive symptoms, a prospective cohort study. Psychological Medicine, 28, 63-79.

Cooper, P., Tomlinson, M., Swartz, L., Woolgar, M., Murray, L., \& Molteno, C. (1999). Post-partum depression and the mother-infant relationship in a South African peri-urban settlement. British Journal of Psychiatry, 175, 554-558.

Cooper, P. J., \& Murray, L. (1998). Postnatal depression. British Medical Journal, 316, 1884-1886.

Cox, J., \& Holden, J. (1996). Perinatal psychiatry: Use and misuse of the Edinburgh postnatal depression scale. London: Gaskell.

Cox, J. L. (1996). Perinatal mental disorder-a cultural approach. International Review of Psychiatry, 8, 9-16.

Gittelsohn, J., Bentley, M. E., Pelto, P. J., Nag, M., Pachauri, S., Harrison, A., \& Landman, L. T., et al. (Eds.). (1994). Listening to women talk about their health: Issues \& evidence from India. New Delhi: Ford Foundation.

Good, B., Good, M. D., \& Moradi, R. (1985). The interpretation of Iranian depressive illness and dysphoric affect. In A. Kleinman, \& B. Good (Eds.), Culture and depression (pp. 369-428). Berkeley: University of California Press.

Healy, D. (1997). The antidepressant era. Cambridge, MA: Harvard University Press.

Howard, R. (1993). Transcultural issues in puerperal mental illness. International Review of Psychiatry, 5, 253-260.

International Institute for Population Sciences (2001). National family health survey II-1998-1999, India. Mumbai, IIPS.

Jadhav, S. (2000). The cultural construction of Western depression. In V. Skultans, \& J. Cox (Eds.), Anthropological approaches to psychological medicine (pp. 41-65). London: Jessica Kingsley Publishers.

Jaswal, S., \& Harpham, T. (1997). Getting sensitive information on sensitive issues: Gynaecological morbidity. Health Policy \& Planning, 12, 173-178.

Kleinman, A. (1987). Anthropology and psychiatry: The role of culture in cross-cultural research on illness. British Journal of Psychiatry, 151, 447-454.

Kumar, R. (1994). Postnatal mental illness: A transcultural perspective. Social Psychiatry \& Psychiatric Epidemiology, 29, 250-264.

Littlewood, R. (1991). From disease to illness and back again. Lancet, 337, 1013-1015.

Nichter, M., \& Nichter, M. (1996). The ethnophysiology and folk dietetics of pregnancy: A case study from south India. In M. Nichter, \& M. Nichter (Eds.), Anthropology \& health: Asian case studies (pp. 35-70). Amsterdam: Gordon \& Breach Science Publishers.

O'Hara, M., \& Swain, A. (1996). Rates and risk of postpartum depression - a metaanalysis. International Review of Psychiatry, 8, 37-54.

Patel, V., Araya, R., Lewis, G., \& Swartz, L. (2001). Socioeconomic factors and mental health. In Mental health: $A$ call for action by World Health Ministers (pp. 39-56). Geneva: World Health Organisation. 
Patel, V., Pereira, J., Coutinho, L., \& Fernandes, R. (1997). Is the labeling of common mental disorders as psychiatric illness useful in primary care? Indian Journal of Psychiatry, 39, 239-246.

Patel, V., Pereira, J., \& Mann, A. (1998). Somatic and psychological models of common mental disorders in India. Psychological Medicine, 28, 135-143.

Patel, V., \& Prince, M. (2001). Aging and mental health in developing countries: Who cares? Qualitative studies from Goa, India. Psychological Medicine, 31, 29-38.

Patel, V., Rodrigues, M., \& De Souza, N. (2002). Gender, poverty \& post-natal depression: A cohort study from Goa, India. American Journal of Psychiatry, 159, 43-47.
Ram, K. (1998). Maternity and the story of enlightenment in the colonies: Tamil coastal women, South India. In K. Ram, \& M. Jolly (Eds.), Maternities \& modernities: Colonial and postcolonial experiences in Asia and the Pacific (pp. 114-143). Cambridge: Cambridge University Press.

Stern, G., \& Kruckman, L. (1983). Multi-disciplinary perspectives on postpartum depression: An anthropological critique. Social Science \& Medicine, 17, 1027-1041.

Winkvist, A., \& Akhtar, H. Z. (2000). God should give daughters to rich families only: Attitudes towards childbearing among low-income women in Punjab, Pakistan. Social Science \& Medicine, 51, 73-81. 